\title{
Impacto del entrenamiento de fuerza en el perfil lipídico de los pacientes con insuficiencia cardiaca. Ensayo clínico aleatorizado (Strong Hearts Trial)
}

\author{
Impact of strength training on the lipid profile of patients with cardiac \\ insufficiency. Randomized clinical trial (Strong Hearts Trial)
}

Javier Eliecer Pereira Rodríguez, ${ }^{*}$ Ximena Velásquez Badillo, ${ }^{\ddagger}$ Devi Geesel Peñaranda Florez, $\$$ Ricardo Pereira Rodríguez, || Pedro Pereira Rodríguez," Miguel Ángel Carranza Castellanos**

\section{Resumen}

Introducción: La insuficiencia cardiaca es la principal causa de muerte en todo el mundo. Objetivo: Conocer el impacto del entrenamiento de fuerza en el perfil lipídico de los pacientes con insuficiencia cardiaca. Material y métodos: Ensayo clínico, aleatorizado de tres años, cuya muestra fue de 920 pacientes con falla cardiaca, los cuales se distribuyeron en tres grupos: sólo ejercicio aeróbico (grupo control [GC]), ejercicio aeróbico más entrenamiento de fuerza de miembros superiores (MMSS) (grupo experimental [GE1]) y ejercicio aeróbico más entrenamiento de fuerza en las extremidades inferiores (MMII) (grupo experimental 2 [GE2]). Se realizaron muestras hematológicas para glucemia y perfil lipídico; también se realizaron pruebas para capacidad aeróbica, frecuencia cardiaca máxima, antropometría, depresión, ansiedad, parámetros clínicos y hemodinámicos. Las pruebas se realizaron antes y después de 24 sesiones de entrenamiento de 60 minutos, tres veces por semana, durante dos meses. Resultados: Al comparar los datos postentrenamiento se obtuvieron resultados favorables en el perfil lipídico del GE1 (Col: $195 \pm 29.5$ vs. $182 \pm 21.4$; Tri: $120 \pm 12.7$ vs. $117 \pm 16.3$; LDL: $116 \pm 23.4$ vs. $109 \pm 12.6)$ y GE2 (Col: $208 \pm 29.6$ vs. $173 \pm 16.5$; Tri: $160 \pm$ 18.2 vs. $138 \pm 7.7$; LDL: $112 \pm 12.7$ vs. $109 \pm 11.6)(p=<0.05)$, además, de todas las variables evaluadas. Conclusiones: El entrenamiento de fuerza combinado con ejercicio aeróbico genera una disminución de los niveles glucémicos y lipídicos significativamente; cabe resaltar que no se encontraron diferencias entre los grupos frente a lipoproteínas de alta densidad.

Palabras clave: Ejercicio, insuficiencia cardiaca, rehabilitación cardiaca, fuerza.

\section{Abstract}

Introduction: Heart failure is the leading cause of death worldwide. Objective: To know the impact of strength training on the lipid profile of patients with heart failure. Material and methods: Randomized controlled trial of 3 years with a sample of 920 patients with heart failure distributed in 3 groups: aerobic Exercise-GC; aerobic exercise plus MMSS training-GE1; aerobic exercise plus MMII training-GE2. Hematological samples were performed to blood glucose levels and lipid profile. In addition, tests and tests for aerobic capacity, maximum heart rate, anthropometry, depression, anxiety, clinical and hemodynamic parameters. The tests are performed before and after 24 training sessions of 60-minute, 3 times a week for two months. Results: When comparing the post data of the training plan, favorable results were obtained in the lipid profile of GE1 (Col: $195 \pm 29.5$ vs $182 \pm 21.4$; Tri: 120 \pm 12.7 vs $117 \pm 16.3$; LDL: $116 \pm 23.4$ vs $109 \pm 12.6$ ) and GE2 (Col: $208 \pm 29.6$ vs $173 \pm 16.5$; Tri: $160 \pm 18.2$ vs $138 \pm 7.7$; LDL: $112 \pm 12.7$ vs $109 \pm 11.6)(p=<0.05)$. In addition, of all the variables evaluated. Conclusions: Strength training combined with aerobic exercise generates a decrease in glycemic and lipid levels; highlighting that no differences were found between the groups versus high density lipoprotein.

Keywords: Exercise, heart failure, cardiac rehabilitation, strength.
* Fisioterapeuta, Especialista en Rehabilitación Cardiopulmonar, Maestrante en Ciencias de la Salud, Maestrante en Innovación Educativa. Universidad Tolteca, Puebla, México.

* Fisioterapeuta, Especialista en Fisioterapia Cardiopulmonar. Clínica San José. Cúcuta-Colombia.

$\S$ Fisioterapeuta, Especialista en Neurorrehabilitación. Magister en dificultades del aprendizaje. Consultorio Independiente Privado, Puebla, México.

"l Médico general. Residente en Medicina de urgencias y cuidado del paciente en estado crítico. Fundación Universitaria de Ciencias de la Salud (FUCS). Bogotá-Colombia.
" Médico general. Unidad de Cuidados Intensivos, Clínica Duarte. Cúcuta-Colombia.

**Estudiante de Fisioterapia, Puebla, México.

Correspondencia:

Javier Eliecer Pereira Rodríguez

Correo electrónico: jepr87@hotmail.com

Aceptado: 21-08-2019. 


\section{INTRODUCCIÓN}

La insuficiencia cardiaca (IC) es considerada por muchos como la patología del milenio, pues su mortalidad va en aumento en la mayoría de países del mundo. La insuficiencia cardiaca puede definirse como un síndrome clínico que se caracteriza por anomalías de la función ventricular y la regulación neurohormonal, que se acompaña de signos de hipertensión venosa pulmonar, sistémica, o de bajo gasto cardiaco, edema, fatiga y disnea que son atribuibles a un daño funcional o estructural de uno o ambos ventrículos, lo cual impide el llenado y vaciado adecuado de las cavidades cardiacas. Esta patología posee como principal característica la progresión en la remodelación desadaptativa del miocardio, si bien puede ser resultado de enfermedades como la hipertensión arterial, diabetes mellitus, infarto agudo al miocardio e incluso, obesidad. ${ }^{1}$

Es fácil darse cuenta de que nos encontramos ante una enfermedad letal que suele afectar, en su mayoría, a personas de edad avanzada, quienes verán disminuida su calidad de vida y supervivencia. ${ }^{2}$ En la actualidad, alrededor de 2 a 3\% del presupuesto sanitario global es destinado al tratamiento de la insuficiencia cardiaca, convirtiéndola en un problema de salud pública. Se ha demostrado que cerca de $2.6 \%$ de la población de Estados Unidos padece insuficiencia cardiaca, con una estimación de 400,000 nuevos casos por cada año. En Europa, en cuanto a la incidencia y prevalencia, los datos epidemiológicos revelan cifras muy cercanas. Si analizamos lo anterior, es importante señalar que cuan mayores sean los índices de envejecimiento poblacional, mayor será la presencia de esta enfermedad, dado que siguen una relación lineal. ${ }^{3}$ En cuanto a la mortalidad, la insuficiencia cardiaca se ha llegado a comparar con los niveles que arroja el cáncer de mama y ovario, es decir, que se encuentra a la altura de los procesos neoplásicos más comunes en la actualidad; ${ }^{4}$ en adición a esto, se sabe que cerca de los cuatro años posteriores al diagnóstico de insuficiencia cardiaca, la mitad de estos pacientes fallecen, es decir, la mortalidad es aún mayor en el primer año. ${ }^{5}$ Por esto es que se le ha denominado como la epidemia cardiovascular del siglo XXI. ${ }^{6}$

Respecto a los factores de riesgo de la insuficiencia cardiaca, la dislipidemia es uno de los principales, ya que contribuye al desarrollo de la enfermedad coronaria. Es importante recordar — a manera de enlace de ideas - el hecho de que un factor importante que desencadena la insuficiencia cardiaca es la obesidad, problema que ha ido en aumento en los últimos años, y que además, el tratamiento utilizado para la disminución del colesterol también genera beneficios cardiovasculares, de ahí su correlación. ${ }^{7}$ Del mismo modo, datos demuestran que el uso de estatinas para el tratamiento de la cardiopatía isqué- mica e hipercolesterolemia tienden a reducir la incidencia de insuficiencia cardiaca; de la misma manera, el uso de atorvastatina ha demostrado una disminución significativa en las hospitalizaciones y mortalidad producida por esta patología. ${ }^{8}$

En relación con los métodos de entrenamiento implementados en la rehabilitación cardiaca, es decir, el conjunto de actividades necesarias para asegurar a los enfermos del corazón una condición física, mental y social óptima que les permita ocupar, por sus propios medios, un lugar normal en la medida de lo posible en la sociedad, hemos de mencionar, por ejemplo, la aplicación de un entrenamiento de fuerza. Este método ha tenido resultados alentadores, pues se tienen registros que, posterior a una dosificación e implementación correcta, individual o grupal, se obtendrán cambios en el porcentaje de masa grasa y la composición corporal, ${ }^{9}$ además de mejorar los niveles de triglicéridos, colesterol total, colesterol de alta y baja densidad, glucosa y proteína C, convirtiéndose así en una herramienta útil y a tener en cuenta para lograr cambios en las enfermedades metabólicas y cardiovasculares. ${ }^{10}$

Ahora bien, hasta hace algunas décadas era del saber popular la idea de que no se podía someter a un paciente con alteraciones del perfil lipídico o cardiaco a un entrenamiento de fuerza, por todos los riesgos que supondría abordar con este tipo de entrenamiento a un paciente con un gran número de factores de riesgo. Sin embargo, estos paradigmas han cambiado, sustentados principalmente por diferentes estudios de buena calidad que resaltan los beneficios fisiológicos, musculoesqueléticos y psicosociales. ${ }^{11-13}$

En la actualidad se sabe que cuando el paciente que sufre de insuficiencia cardiaca es sometido a entrenamiento de fuerza, los síntomas, la capacidad funcional, la función respiratoria, así como en la potencia y la masa muscular mejoran. Por esta razón, se ha recalcado que el ejercicio de elevada intensidad no genera una mala respuesta hemodinámica en el sistema cardiovascular previamente dañado. ${ }^{14}$

Dicho lo anterior, surge como pregunta de investigación: ¿cuál es el impacto del entrenamiento de fuerza en el perfil lipídico de los pacientes con insuficiencia cardiaca? Buscando una posible respuesta a esta pregunta, el objetivo principal de la presente investigación es conocer el impacto del entrenamiento de fuerza en el perfil lipídico de los pacientes con insuficiencia cardiaca.

\section{MATERIAL Y MÉTODOS}

Esta investigación se pensó como un ensayo controlado y aleatorizado, con un muestreo probabilístico básico por medio de una tabla de números aleatorios, cuyo orden se aleatorizó a través del programa Microsoft Excel 16.0. 
El estudio se realizó con una muestra conformada por 920 pacientes de rehabilitación cardiaca en Colombia, quienes al final, de acuerdo con los criterios de exclusión, quedaron 764 individuos. Éstos se organizaron en tres grupos (Figura 1).

El grupo control al final resultó de 253 participantes, quienes se seleccionaron a través de los registros médicos desde el año 1980 (ejercicio aeróbico sin entrenamiento de fuerza): 256 participantes para el grupo experimental 1 (GE1: ejercicio aeróbico más entrenamiento de fuerza en las extremidades superiores [MMSS]) y el grupo experimental 2, con 255 (GE2: ejercicio aeróbico más entrenamiento de fuerza para las extremidades inferiores [MMII]). Cabe resaltar que el presente artículo se da como resultado del macroproyecto Strong Hearts Trial, incluido en el sistema de registro y resultados del protocolo ClinicalTrials.gov de la National Library of Medicine (NLM), del National Institutes of Health $(\mathrm{NIH})$ y de la Food and Drug Administration (FDA): NCT03913780. La presente investigación se llevó a cabo en un periodo de tres años (de abril de 2014 a 2017), la cual contiene las siguientes particularidades:

Características de los participantes. Los participantes contenían características similares desde el punto de vista de la fracción de eyección, la clase funcional, la glucosa, el perfil lipídico, el porcentaje muscular, la grasa y el IMC (índice de masa corporal), la circunferencia abdominal, el sobrepeso, la obesidad, la prevalencia de diabetes, la hipertensión (hipertensión), la enfermedad renal, los factores de riesgo cardiovascular y el procedimiento quirúrgico cardiovascular para su respectivo análisis.
Inscripción

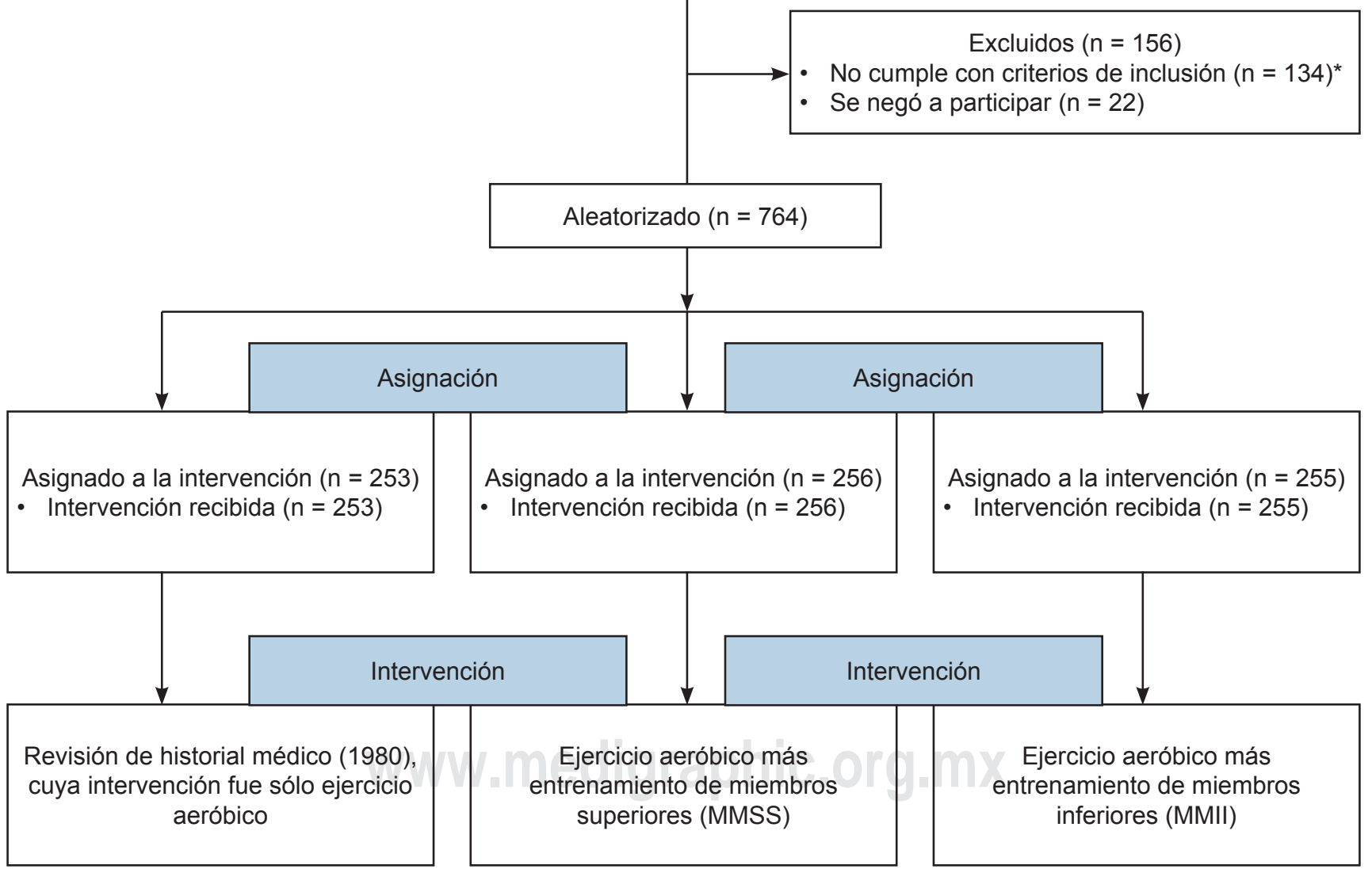

*134 pacientes excluidos: siete debido a enfermedades infecciosas, siete por tromboflebitis, nueve por angina inestable, nueve por descompensación diabetes, 21 debido a hipertensión sistólica $>190 \mathrm{mmHg}, 22$ referían una dolorosa safenectomía que impidió las pruebas y 59 pacientes con insuficiencia cardiaca descompensada.

Figura 1: Diagrama de flujo de distribución de pacientes. 
Criterios de inclusión. Los participantes que se incluyeron fueron aquéllos sometidos a una cirugía cardiovascular y que también hubieran asistido a un programa de rehabilitación cardiaca (RHC) fase II. También, como requisito indispensable, debían firmar el consentimiento informado, previamente avalado por el Comité de Ética e Investigación de la institución. De la misma manera, se tomaron en consideración aspectos como que el paciente tuviera insuficiencia cardiaca, y que no presentara alguna dificultad al realizar las pruebas, medidas o cuestionarios necesarios para la investigación y, por supuesto, se reiteró que debía acudir tres veces por semana al RHC.

Criterio de exclusión. Se optó por excluir a aquellos pacientes que padecieran de angina inestable, dolor en el miembro inferior, que durante el reposo reflejaran una frecuencia cardiaca en reposo $>120$ latidos por minuto, una presión arterial sistólica $>190 \mathrm{mmHg}$, presión arterial diastólica $<120 \mathrm{mmHg}$. En esta misma sintonía, se dejó fuera a aquellos pacientes que tuvieran contraindicado el acudir a rehabilitación cardiaca (Tabla 1). Hecha esta salvedad, se le reiteró al paciente que si tenía la intención de abandonar la investigación, lo podía hacer en el momento en que lo deseara.

\section{Medidas antropométricas}

Se recopiló en cada uno de los pacientes la siguiente información: talla, peso, circunferencia abdominal, índice de masa corporal y porcentaje de grasa y músculo. Lo anterior se tomó a partir de referencias técnicas estandarizadas en la población colombiana. En relación al porcentaje de grasa, peso y músculo se recabaron con la balanza digital Tezzio TB-30037, la cual se calibró y colocó en una superficie estable y plana, cumpliendo así con lo señalado en el manual del usuario.

En lo que respecta a la talla, ésta se obtuvo a través de un Kramer 2104 Adult Acrylic, para lo cual se pidió al paciente colocarse en posición bípeda, manteniendo la cabeza en plano de Frankfort, con las extremidades inferiores (MMII) por completo pegadas a la pared y, a fin de evitar la lordosis, se pidió mantener los hombros lo más relajados que le fuera posible. Llegados a este punto, con base en estas variables, se determinó el IMC en kg/ $\mathrm{m}^{2}$. Posteriormente, con ayuda de una cinta métrica y de precisión de $1 \mathrm{~mm}$, se obtuvo la circunferencia abdominal, respetando las referencias anatómicas plasmadas por Frisancho. ${ }^{15}$ La interpretación fue mediante los «puntos de corte de perímetro de cintura para el diagnóstico de obesidad abdominal en población colombiana, usando bioimpedanciometría como estándar de referencia», siguiendo a Buendía R y colaboradores, de $91 \mathrm{~cm}$ para hombres y de $89 \mathrm{~cm}$ para mujeres. ${ }^{16}$
Tabla 1: Contraindicación de la rehabilitación cardiaca.

IAM precoz

Angina inestable

Valvulopatía severa

Insuficiencia cardiaca descompensada

Condición ortopédica severa que impide la realización de ejercicios

Arritmias ventriculares complejas

Sospecha de lesión de tronco de la arteria coronaria izquierda

Obstrucción sintomática severa del tracto de salida del ventrículo izquierdo

Endocarditis infecciosa

Hipertensión arterial descompensada: PAS > 190 mmHg y/o $\mathrm{AD}>120 \mathrm{mmHg}$

Hipotensión ortostática por encima de $20 \mathrm{mmHg}$ con síntomas

Tromboembolismo pulmonar y tromboflebitis

Disección de aneurisma aórtico

Endocarditis infecciosa

Enfermedad cardiaca congénita grave no corregida

Bloqueo atrioventricular no corregido de 3er. grado

Diabetes descompensada

Otras afecciones metabólicas como tiroiditis aguda, hipopotasemia, hipercalemia o hipovolemia

IAM: infarto agudo al miocardio; PAS: presión sanguínea sistólica; PAD: presión arterial diastólica.

\section{Parámetros clínicos y hemodinámicos}

Dicho lo anterior, es importante señalar que durante el primer y último día de la sesión de entrenamiento se tomaron los niveles de glucosa en sangre en ayuno. También entre las 7:00 y 8:00 a. m., posterior a un ayuno de ocho a 10 horas, se recolectaron las muestras de sangre. Cabe recalcar que dichas muestras fueron analizadas en el laboratorio clínico del hospital, y que además sirvió para determinar los niveles de triglicéridos, colesterol, lipoproteínas de baja densidad (LDL) y lipoproteínas de alta densidad (HDL). Del mismo modo, a cada participante se le sometió, previo y posteriormente al programa de entrenamiento, a una ecocardiografía 2-D (bidimensional), con la finalidad de analizar la movilidad en tiempo real, fracción de eyección del ventrículo izquierdo $y$, en general, visualizar las estructuras cardiacas. Durante este mismo procedimiento, se asignó al paciente dentro de la clasificación de la NYHA (New York Heart Association), la cual consta de cuatro clases (I, II, III y IV).

Del mismo modo, el esfuerzo y la disnea percibida fueron evaluadas por medio de la escala de Borg modificada. ${ }^{17}$ La frecuencia cardiaca fue detectada por el Polar Multisport RS800CX system; la frecuencia respiratoria, al igual que la presión arterial sistólica y diastólica, se 
obtuvieron manualmente. Por su parte, la saturación de oxígeno se obtuvo con un oxímetro portátil (Nellcor Puritan Bennett).

\section{Pruebas y cuestionarios}

Con la intención de conocer el estado inicial del paciente, se solicitó al Área de Rehabilitación Cardiaca realizar una evaluación médica, rescatando la información sobre aspectos sociodemográficos, antropométricos y fisiológicos. Posteriormente, se realizó la prueba de caminata de seis minutos, a fin de obtener los datos relacionados con la capacidad funcional; dicha prueba se realizó antes y después del programa de rehabilitación cardiaca. El protocolo de la prueba de caminata de seis minutos se realizó de acuerdo con la ATS Statement: Guidelines for the six-minute walk test de la American Thoracic Society. ${ }^{18,19}$ El cuestionario de una repetición máxima (1RM) se utilizó para obtener el peso al inicio del entrenamiento de fuerza, tanto en las extremidades superiores (MMSS) como en las extremidades inferiores (MMII).

Se dio por aceptable la repetición máxima cuando ésta fuera realizada sin compensaciones de la musculatura aledaña y cuando el músculo valorado realizara un recorrido completo. Al día siguiente de las evaluaciones, se pidió al paciente volver para realizar una prueba de esfuerzo según el protocolo Naughton, recomendado en pacientes de alto riesgo. Es importante mencionar que para dicha prueba se informó al paciente que debía abstenerse de ingerir bebidas alcohólicas, fumar, ingerir algún medicamento o sustancia que pudiese generar alteraciones de sus signos vitales o rendimiento durante la prueba.

\section{Fuerza}

La fuerza fue valorada mediante dinamometría con el Hand Grip CAMRY electronic hand dynamometer model EH101. Para esta prueba, el sujeto tenía que permanecer de pie o sentado en una posición cómoda y sin apoyabrazos, con los hombros aducidos y sin rotación; y el codo debía estar flexionado a $90^{\circ}$, con los antebrazos y muñecas en posición neutra.

Se midió la fuerza de prensión en la mano hábil y se registraron tres determinaciones consecutivas, respetando el tiempo de recuperación muscular, que es de aproximadamente un minuto. Asimismo, para conocer el peso inicial del entrenamiento de fuerza para las extremidades superiores (MMSS) e inferiores (MMII) se consideró apropiada la prueba de una repetición máxima (1RM), con una extensión completa del grupo muscular utilizado y sin sustituciones musculares.

\section{Asignación de la muestra}

Esta investigación abarcó una población total de 764 participantes (sexo masculino de 433 en comparación con el sexo femenino de 331), los cuales se dividieron en tres grupos de una manera eventual, con un modelo probabilístico simple mediante una tabla de números aleatorios, cuya aleatorización se llevó a cabo mediante el programa Microsoft Excel 16.0.

\section{Metodología ciega}

Se efectuó un estudio clínico simple a ciegas, para lo cual los participantes fueron analizados por un profesional ajeno a la investigación (fisiatra del Servicio de Rehabilitación Cardiaca), y también con un análisis de sangre en el laboratorio. El paso siguiente consistió en ingresar a los pacientes en una base de datos de Microsoft Excel 16.0, asignándoles únicamente un número de identificación, logrando así cegar a los autores. La distribución aleatoria fue realizada por un profesional en ingeniería de sistema de la institución y externo al grupo de autores e investigadores del macroproyecto Strong Hearts Trial.

Referente al grupo control, éstos fueron seleccionados de manera aleatorizada de igual forma que a los GE1 y GE2 de todos los pacientes intervenidos en el año de 1980. Posterior a la selección, el autor principal (mencionado a partir de aquí con sus iniciales: JPR) revisó que los registros médicos seleccionados poseyeran la información requerida para así poder determinar los cambios postentrenamiento y las variables que se evaluarían tanto en el grupo experimental 1 como en el grupo 2.

Al inicio, y una vez concluidas las 24 sesiones del entrenamiento según el grupo asignado, se realizaron las pruebas y cuestionarios para posteriormente mostrar un informe minucioso de los cambios fisiológicos postentrenamiento, así como del comportamiento a lo largo de cada una de las sesiones del programa. Cabe recalcar que desde el inicio de las pruebas, así como al final de la capacitación, no se estableció una conversación fuera de contexto entre pacientes e investigadores. Únicamente el autor fue quien se reunió de manera periódica con los terapeutas cardiopulmonares, con la intención de verificar y armonizar la intervención en cada uno de los grupos, todo ello sin intimar con los participantes, intervención o registro.

Teniendo en cuenta la información recopilada antes y después, los análisis estadísticos para el grupo experimental 1 se llevaron a cabo por los autores JPR y XVB; para el grupo experimental 2 por los autores PPR y DPF, y para el grupo de control por los autores MCC y RPR. Finalmente, una vez que las diferentes variables se estudiaron de manera cegada, todos los autores fueron informados sobre los gru- 
pos con sus participantes correspondientes y los resultados para producir las conclusiones.

\section{Intervención por grupo}

El programa de rehabilitación cardiaca consistió en 24 sesiones de 70 minutos al día (10 minutos de calentamiento, 50 minutos de entrenamiento y 10 minutos de enfriamiento), tres veces a la semana durante dos meses. Con respecto al calentamiento, se basó en ejercicios de movilización por grupos musculares. El enfriamiento se realizó con ejercicios de propiocepción, coordinación, estiramientos y ejercicios de respiración.

En relación con los 50 minutos de entrenamiento, se modificó de acuerdo con cada grupo asignado. Para el grupo control (registros médicos), sólo se realizó ejercicio aeróbico en una banda sin fin, recumbent (bicicleta reclinable), remo y bicicleta elíptica. En el grupo experimental 1, se realizó ejercicio aeróbico (como el grupo control) más ejercicios de fuerza para las extremidades superiores con mancuernas, equipos de multifuerza y TheraBand (liga de resistencia). Para el entrenamiento del grupo experimental 2 , éste consistió en el mismo orden que el grupo control más el entrenamiento de fuerza para las extremidades inferiores con equipos y ejercicios en multifuerza para la activación de la bomba de soleo gemelar.

Respecto a la prescripción del ejercicio aeróbico, según el protocolo de Naughton, fue de 50 a $80 \%$ de su frecuencia cardiaca máxima (FCM) alcanzada en la prueba de esfuerzo. Para la fuerza, se determinó entre 30 y $50 \%$ de 1 RM. Para los grupos 1 y 2, el entrenamiento de fuerza se basó en un aumento progresivo de la carga, según Kraemer y Ratamess (2005).

\section{CONSIDERACIONES ÉTICAS}

Es importante recalcar que tanto el desarrollo y el diseño de la presente investigación se diseñó bajo los parámetros éticos de la Declaración de Helsinki y la Resolución núm. 008430 del Ministerio de Salud de Colombia, con la corroboración por parte de directivos y del Comité de Ética de la institución donde se realizó la investigación.

\section{Análisis estadístico}

Se realizó una base de datos en Microsoft Excel 16.0 con todos los individuos, sus resultados de las pruebas, cuestionarios y preentrenamiento y postentrenamiento. Luego, se efectuaron las estadísticas descriptivas para evaluar y señalar los datos por promedios con su correspondiente desviación estándar. La normalidad de los datos se evaluó mediante la prueba de Kolmogorov-
Smirnov, y la indicación de especificidad fue evidente para todos los análisis. Asimismo, a través de la prueba de Tukey se utilizó el análisis de varianza ANOVA (análisis de varianza de una vía) y posteriormente se realizaron las pruebas post hoc para evaluar las características de los diferentes grupos de edad, género y antropometría. En todos los casos se estableció un nivel de significación de $5 \%(p<0.05)$. Todo lo anterior se llevó a cabo en el programa Stata: Software for Statistics and Data Science.

\section{RESULTADOS}

El presente ensayo clínico aleatorizado inició con una muestra total de 920 pacientes entrevistados en rehabilitación cardiaca, de los cuales 253 fueron de expedientes médicos. Posteriormente a los filtros, se contó con la participación de 764 pacientes, de los cuales 433 fueron hombres y 331 mujeres. Las características de la población de estudio se muestran en la tabla 2.

En relación con los procedimientos quirúrgicos cardiovasculares a los que pudieron ser sometidos los participantes, la revascularización miocárdica ocupó el primer lugar con $44.10 \%$, seguido de angioplastia con $32.72 \%$, reemplazo valvular con $12.17 \%$, cierre de comunicación interauricular con $4.71 \%$, cirugía de Bentall con $4.97 \%$ y trasplante de corazón con 1.3\% (Figura 2).

Al comparar los valores de fracción de eyección y consumo máximo de oxígeno $\left(\mathrm{VO}_{2}\right)$ como indicador de tolerancia al ejercicio y los metros recorridos antes y después de la intervención de cada programa de entrenamiento basado en el ejercicio aeróbico más un componente de fuerza muscular, se observó una mejoría significativa en todas las variables ( $\mathrm{p}<0.05 \%)$. Además, al comparar los resultados entre los grupos, se logró evidenciar mejores resultados con diferencia significativa en el grupo experimental 2 (ejercicio aeróbico más entrenamiento de fuerza en MMII) $(p>0.05 \%)$ y el grupo control $(p<0.05 \%)$. En cuanto a los datos antes y después de medir peso, índice de masa corporal, circunferencia abdominal y porcentaje graso, se observó una disminución significativa; caso contrario a lo que sucedió con el porcentaje muscular y la fuerza prensil, parámetros que registraron un aumento significativo entre las evaluaciones.

Se demostró una diferencia significativa en las mediciones antes y después, de manera individual en cada grupo $(p \leq 0.05)$; al momento de compararlos entre ellos, no se encontró diferencia en la variable de HDL ( $p>0.05)$. En lo que se refiere a la calidad de vida, los datos revelan una mejora en relación con la evaluación inicial y final (GC: 5.6 \pm 16.3 vs. $49.1 \pm 8.8$; GE1: $50 \pm 12$ vs. $40.5 \pm 4.5$; GE2: $59 \pm 7.5$ vs. $34.5 \pm 6.9$ ) (Tabla 3). 
Tabla 2: Características iniciales de la población de estudio $(n=764)$.

Grupo control $(n=253)$

Grupo experimental $1(n=256)$

Grupo experimental $2(n=255)$
Características

Género

Edad (años)

Fracción de eyección (\%)

Altura $(\mathrm{m})$

Peso $(\mathrm{kg})$

IMC

Circunferencia abdominal $(\mathrm{cm})$

Porcentaje de grasa $(\%)$

Porcentaje de músculo (\%)

Colesterol total $(\mathrm{mg} / \mathrm{dL})$

Triglicéridos ( $\mathrm{mg} / \mathrm{dL})$

$\mathrm{HDL}(\mathrm{mg} / \mathrm{dL})$

Glucosa (mg/dL)

VO2 (mL/kg-1/min-1)

Distancia recorrida $(\mathrm{m})$

FCM en prueba de esfuerzo (Ipm)

Fuerza prensil (kg)

Calidad de vida

Sobrepeso u obesidad (IMC) (\%)

Obesidad abdominal (\%)

Dislipidemia (\%)

Hipertensión arterial (\%)

Diabetes mellitus (\%)

Enfermedad renal (\%)

Sedentarismo (\%)

Depresión (\%)

Ansiedad (\%)

Tabaquismo (\%)

Alcoholismo (\%)

Dieta inadecuada (\%)

Historial de IAM (\%)

Género femenino (\%)

Edad $^{\dagger}$
LDL (mg/dL)

$\begin{array}{ccc}\text { M: } 144 ; \mathrm{F}: 109 & \text { M: } 155 ; \mathrm{F}: 101 & \text { M: } 134 ; \mathrm{F}: 121 \\ 65 \pm 3 & 67 \pm 8 & 64 \pm 5 \\ 39 \pm 3.1 & 40 \pm 2.6 & 40 \pm 3.5 \\ 1.68 \pm 17.8 & 1.60 \pm 12 & 1.64 \pm 15.4 \\ 75 \pm 18.3 & 80 \pm 14.9 & 79 \pm 11.6 \\ 29 \pm 3.9 & 32 \pm 3.1 & 31 \pm 4.6 \\ 89 \pm 7.2 & 90 \pm 6.5 & 93 \pm 9.7 \\ 27 \pm 6.4 & 25 \pm 2.4 & 29 \pm 4.3 \\ 29 \pm 15.6 & 32 \pm 9.1 & 30 \pm 12.9 \\ 211 \pm 32.3 & 195 \pm 29.5 & 208 \pm 29.6 \\ 143 \pm 21.2 & 120 \pm 12 & 160 \pm 18.2 \\ 149 \pm 15.4 & 116 \pm 23.4 & 112 \pm 12.7 \\ 45 \pm 8.2 & 41 \pm 2.6 & 45 \pm 9.3 \\ 127 \pm 6.1 & 131 \pm 11.5 & 124 \pm 9.5 \\ 8.9 \pm 2.1 & 7.3 \pm 5.7 & 7.8 \pm 4.3 \\ 223 \pm 38 & 243 \pm 23 & 219 \pm 53 \\ 152 \pm 13 & 146 \pm 16 & 148 \pm 12 \\ 24 \pm 7.6 & 23 \pm 10.1 & 26.7 \pm 4.2 \\ 54.6 \pm 16.3 & 50 \pm 12 & 59 \pm 7.5 \\ 74 & 78 & 81 \\ 83 & 88 & 89 \\ 63 & 57 & 49 \\ 79 & 78 & 82 \\ 54 & 60 & 57 \\ 13 & 9 & 11 \\ 93 & 91 & 95 \\ 23 & 21 & 27 \\ 18 & 11 & 15 \\ 81 & 76 & 86 \\ 33 & 13 & 20 \\ 44 & 56 & 30 \\ 90 & 89 & 93 \\ 43 & 39 & 47 \\ 92 & 91 & 94\end{array}$

HDL: lipoproteínas de alta densidad; LDL: lipoproteínas de baja densidad; IMC: índice de masa corporal; VO2: consumo máximo de oxígeno; FCM: frecuencia cardiaca máxima; kg: kilogramos; mg/dL: miligramos por decilitro; $\mathrm{cm}$ : centímetros; m: metros. IAM: infarto agudo al miocardio.

${ }^{\dagger}$ Mujer > 65 años y hombre > 40 años, según la Rev Colombia Cardiol. 2011; 18 (4): 177-182.

\section{DISCUSIÓN}

Dentro de los programas de rehabilitación cardiaca, el entrenamiento de fuerza mejora la tolerancia al ejercicio de una manera segura y sin efectos adversos significativos. ${ }^{20}$ Con la intención de lograr mejoras sistemáticas, diversas modalidades de entrenamiento han sido adoptadas, y en este caso, especialmente del perfil lipídico, se sugiere que deportes mixtos de alto componente dinámico y contracciones musculares con alto impacto articular y muscular, y que sean practicados a alta intensidad, pueden presentar un perfil lipídico desfavorable. ${ }^{21}$

La bibliografía actual señala que el entrenamiento de fuerza tiene efectos positivos en la reducción o normalización de los valores del perfil lipídico, siendo éste un factor de morbilidad importante. ${ }^{22,23}$ Resaltando con énfasis lo que menciona Dure y Viñas ${ }^{24}$ respecto a que realizar un ejercicio físico programado, dirigido y bien ejecutado contribuirá a lo anterior de mejor manera. Tal como la 
metodología planteada y los resultados obtenidos en la presente investigación.

En un programa de intervención con características similares a nuestro estudio, con una duración de tres meses, Zapata y colaboradores ${ }^{25}$ utilizaron un programa de ejercicio de sobrecarga, donde aislaron los segmentos musculares durante un minuto, por dos minutos de descanso, y repitiendo en tres ocasiones; con ello lograron disminuir en el grupo experimental los niveles de colesterol total y LDL, con un aumento del HDL; caso similar a lo obtenido en el presente estudio, especulando así que a mayor duración del programa, mayor relación con las mejoras del perfil lipídico.

Por otra parte, Rosello y su equipo de investigación ${ }^{26}$ señalan que algunos pacientes, previo a iniciar el programa de rehabilitación, pudieran haber adoptado cambios nutricionales que provocaron cambios, logrando así modificaciones en el perfil lípido del IMC. Si bien los resultados en nuestro estudio y en los mencionados anteriormente son favorables, es necesario, como lo menciona Wong y otros, ${ }^{27}$ encaminar al paciente hacia una educación nutricional, estando dentro del grupo tratado y que ésta continúe de manera individual para lograr así generar hábitos alimenticios adecuados.

Ahora bien, resulta fundamental el hecho de fomentar la aplicación de un programa de ejercicio terapéutico en este tipo de población, haciéndolo parte de los beneficios que éste trae consigo, ya que se ha demostrado que tiene efectos positivos cuando se aplica desde el centro de intervención primaria como una herramienta terapéutica y a su vez preventiva, concluyendo que las personas más beneficiadas de este tipo de intervención serán aquéllas que padezcan de dislipidemias. ${ }^{28}$
En relación con los datos favorables de nuestro estudio, tenemos a Oviedo y su grupo, ${ }^{23}$ quienes en su investigación realizaron el trabajo de fuerza a través de bandas TheraBand. Los participantes ejecutaban ejercicios para miembros superiores e inferiores dentro un mismo circuito, obteniendo resultados estadísticamente significativos en los niveles del perfil lipídico y la presión arterial; punto importante al tratarse de una población de estudios con alteraciones cardiacas. Ahora bien, cabe resaltar que los niveles de colesterol HDL no presentaron diferencias entre los grupos, pero sí al comparar los cambios postentrenamiento de manera individual, debido a que la evidencia nos muestra que el efecto del ejercicio aeróbico, específicamente en el colesterol HDL, aumenta de forma modesta en comparación con los triglicéridos, colesterol e inclusive el LDL. Además, es importante resaltar que nuestros resultados referentes al HDL concuerdan con los 25 ensayos clínicos aleatorizados que se han incluido en un metaanálisis, ${ }^{29}$ cuya finalidad fue determinar el efecto del ejercicio en el colesterol HDL. Dichos resultados mencionan que el colesterol HDL incrementa en promedio 2.65 $\mathrm{mg} / \mathrm{dL}$, y que este aumento genera un gasto calórico promedio mínimo $900 \mathrm{Kcal}$ por semana o 120 minutos de ejercicio semanales. Además, se pudo concluir que, por cada 10 minutos que se prolongue el ejercicio, hay un incremento adicional de colesterol HDL de $1.4 \mathrm{mg} /$ $\mathrm{dL}$, lo cual concuerda con los resultados presentados por Kelley GA y su grupo ${ }^{30}$ y, en cierta medida, con los nuestros debido a que el aumento no fue de $2.6 \mathrm{mg} / \mathrm{dL}$, sino mucho mayor que el referido (GC: $3.2 \pm 4.1$; GE1: $6 \pm 1.2$; GE2: $5 \pm 7.0$ ) por estos estudios.

Figura 2: Procedimientos quirúrgicos.

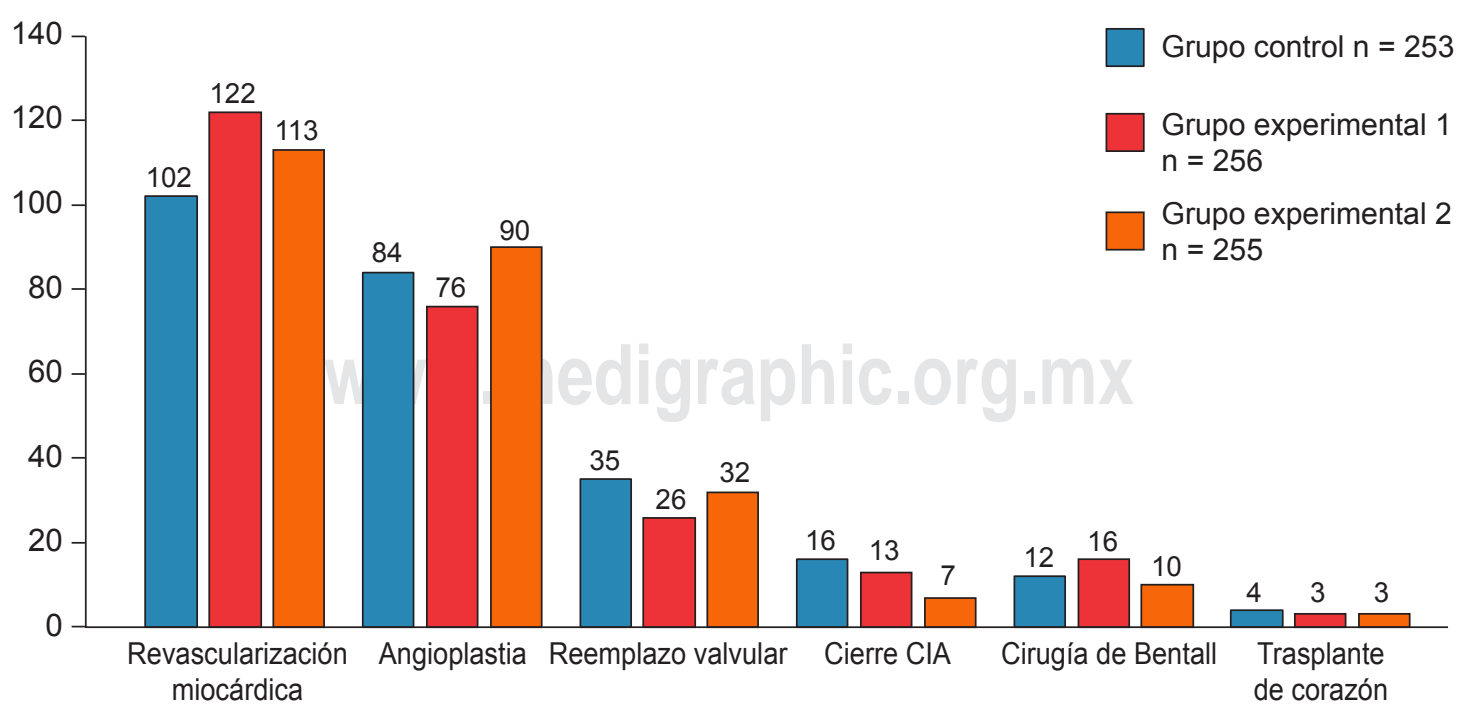




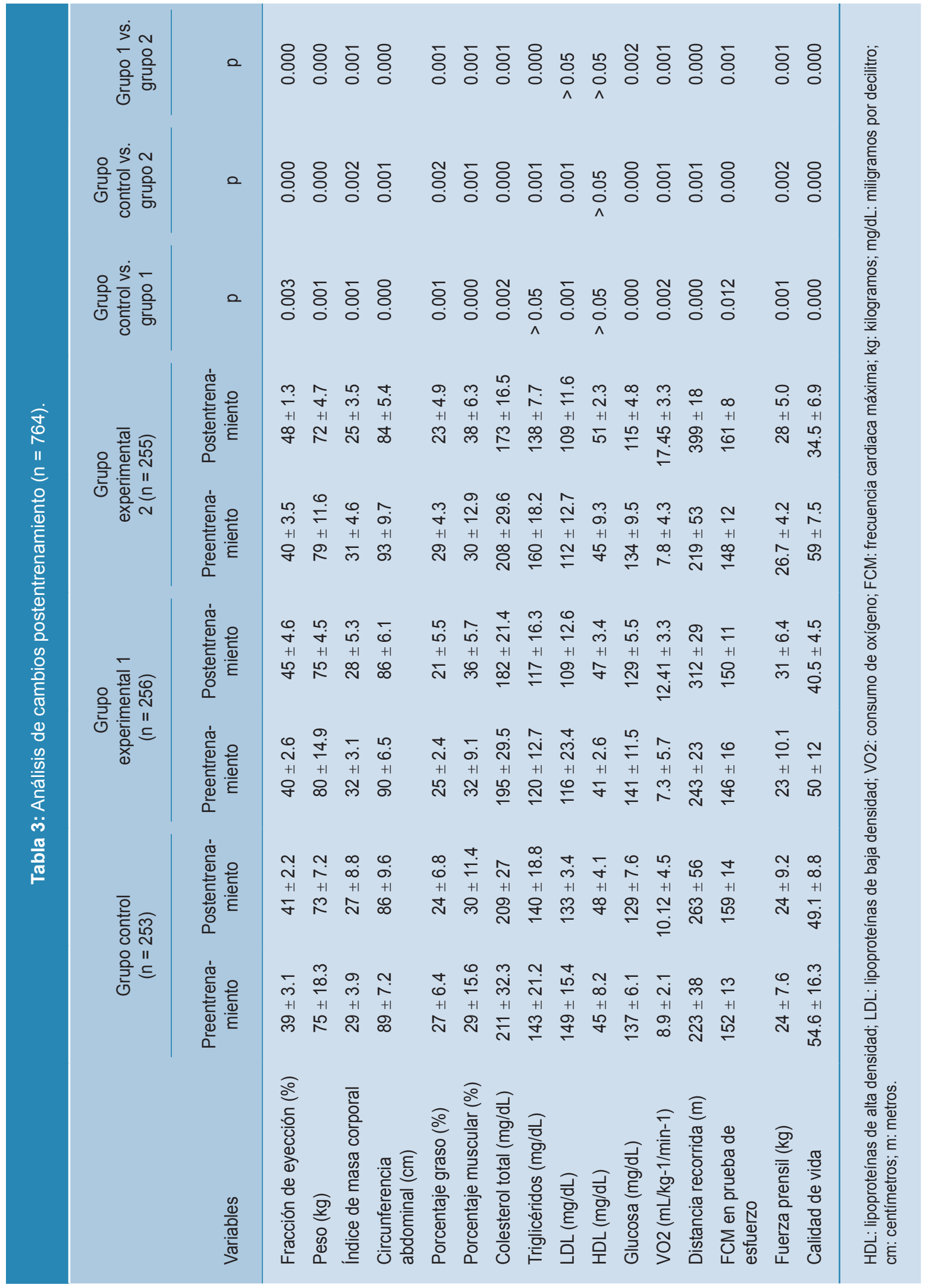




\section{LIMITACIONES}

En esta clase de proyectos con múltiples variables asociadas con una patología, como la insuficiencia cardiaca, que presenta un conjunto de comorbilidades, es de vital importancia analizar el tratamiento farmacológico y la dieta seguida por cada uno de los participantes. Situaciones que no fueron tomadas en cuenta, pero inevitablemente cumplen un factor predominante en la respuesta cardiovascular al ejercicio. Además, es de resaltar que la presente investigación utilizó la prueba de caminata de seis minutos para determinar los valores de $\mathrm{VO}_{2}$, pero si es posible, una ergoespirometría o un análisis de gases sería ideal para la investigación.

\section{CONCLUSIONES}

El entrenamiento de fuerza combinado con el ejercicio aeróbico genera una disminución de los niveles glucémicos y lipídicos significativamente después de 24 sesiones de entrenamiento, logrando demostrar diferencias significativas frente al grupo control. Además, se observan datos significativos de disminución en aspectos como el peso, índice de masa corporal, circunferencia abdominal, porcentaje graso y aumento de fracción de eyección, fuerza prensil, distancia recorrida, frecuencia cardiaca máxima, porcentaje muscular y calidad de vida.

\section{REFERENCIAS}

1. Pereira-Rodríguez JE, Rincón-Gonzales G, Niño-Serrato DR. Insuficiencia cardiaca: Aspectos básicos de una epidemia en aumento. Cor Salud. 2016; 8 (1): 58-70.

2. Cagide A. Evolución del tratamiento de la insuficiencia cardiaca. Insuf Card. 2015; 10: 49-55.

3. Farmakis D, Parissis J, Lekakis J, Filippatos G. Insuficiencia cardiaca aguda: epidemiología, factores de riesgo y prevención. Revista Española de Cardiología. 2015; 68 (3): 245-248. doi:10.1016/j. recesp.2014.11.009

4. Sánchez J, López A. Insuficiencia cardiaca. Generalidades. Medicine. Programa de Formación Médica Continuada Acreditado. Medicine. 2017; 12 (35): 2085-2091. doi:10.1016/j.med.2017.06.001.

5. Aguilar J. C. La insuficiencia cardiaca en el siglo XXI. Epidemiología y consecuencias económicas. Cirugía Cardiovascular. 2011; 18 (2): 83-89.

6. Ortiz G, De la Cruz J, Camacho G, Vega L, Pérez H, Larrañaga E. Guía de Tratamiento Farmacológico de dislipidemias para el primer nivel de atención. Rev Mex Cardiol. 2013; 24 (3): 103-129.

7. Pallarés V, Pascual V, Godoy D. Dislipidemia y riesgo vascular. Una revisión basada en nuevas evidencias. Medicina de Familia. 2015; 41 (8): 435-445.

8. Costa S, Reina-Couto M, Albino-Teixeira A, Sousa, T. Statins and oxidative stress in chronic heart failure. Revista Portuguesa de Cardiologia. 2016; 35 (1): 41-57. doi:10.1016/j.repce.2015.12.016

9. Ribeiro A, Tomeleri C, Souza F, Pina F, Schoenfeld B, Nascimento, $M$ et al. Effect of resistance training on $\mathrm{C}$-reactive protein, blood glucose and lipid profile in older women with differing levels of RT experience. AGE. 2015; 37 (6). doi:10.1007/s11357-015-9849-y

10. Hernández S, Mustelier J, Larrinaga V, Rodríguez L, Sorio B, Peña V et al. Efecto del entrenamiento físico en pacientes con insuficiencia cardiaca crónica y fracción de eyección del ventrículo izquierdo deprimida. Revista Cubana de Cardiología y Cirugía Cardiovascular. 2018; 24: 3.

11. Pina IL, Apstein C, Balady G, Romualdo B, Chaitman B et al. Exercise and heart failure: a statement from the American Heart Association Committee on exercise, rehabilitation, and prevention. Circulation. 2003; 107: 1210-25. DOI:10.1161/01.cir.0000055013.92097.40.

12. Fleg J, Cooper, L, Borlaug, BA, Haykowsky MJ, Kraus WE, Levine B. Exercise training as therapy for heart failure: current status and future directions. Circulation: Heart Failure. 2015; 8 (1): 209-220. DOI:10.1161/circheartfailure.113.001420

13. Pozo P, González G. La prescripción del ejercicio de fuerza en la insuficiencia cardiaca crónica: una revisión del estado actual de la situación. Enfermería en Cardiología. Enferm Cardiol. 2012; 55-56: 17-21.

14. Perez-Gómez J, Vicente-Rodríguez G, Ara I, Martínez-Redondo D. Puzo J, Moreno L et al. Effect of endurance and resistance training on regional fat mass and lipid profile. Nutr Hosp. 2013; 28 (2): 340-346. doi:10.3305/nh.2013.28.2.6200.

15. Frisancho R. Anthropometric standard for the assessment of growth and nutritional status. Ann Arbor: University of Michigan Press; 1993; pp. 9-31.

16. Buendía R, Zambrano M, Díaz Á, Reino A, Ramírez J, Espinosa E. Puntos de corte de perímetro de cintura para el diagnóstico de obesidad abdominal en población colombiana usando bioimpedanciometría como estándar de referencia. Revista Colombiana de Cardiología. 2016; 23 (1): 19-25. doi:10.1016/j. rccar.2015.07.011

17. Fett CA, Fett WCR, Marchini JS. Circuit weight training vs jogging in metabolic risk factors of overweight/obese women. Arq Bra Cardiol. 2009; 93: 519-25. doi:10.1590/s0066-782×2009001100013.

18. Enright $P$, Sherrill D. Reference equations for the six-minute walk in healthy adults. Am J Respir Crit Care Med. 1998; 158: 1384-1387.

19. American Thoracic Society. ATS statement: guidelines for the sixminute walk test. Am J Respir Crit Care Med. 2002; 166: 111-117.

20. Velasco J, Cosín J, Maroto J, Muñiz J, Casasnova J, Plaza I et al. Guías de práctica clínica de la Sociedad Española de Cardiología en prevención cardiovascular y rehabilitación cardiaca. Revista Española de Cardiología. 2000; 53 (8): 1095-1120.

21. Boratia A. La práctica deportiva mejora el perfil lipídico plasmático, pero ża cualquier intensidad? Rev Esp Cardiol. 2004; 57 (6): 495-498. doi:10.1157/13062914.

22. Kelley G, Kelley K, Franklin B. Aerobic exercise and lipids and lipoproteins in patients with cardiovascular disease: a metaanalysis of randomized controlled trials. Journal of Cardiopulmonary Rehabilitation. 2006; 26 (3): 131.

23. Oviedo R, Niño O, Bellomío C, Gonzalez D. Guerra, M. Entrenamiento, presión arterial y lípidos en adultos con prehipertensión, RETOS. Nuevas Tendencias en Educación Física, Deporte y Recreación. 2015; 27: 67-72.

24. Dure $M$, Viñas L, Quintana A. La actividad física como vía para disminuir el riesgo de la cardiopatía isquémica. Revista Digital Buenos Aires. 2011; 16 (162)

25. Zapata-Lamana, R, Cigarroa, I, Díaz, E, Saavedra, C. Reducción del riesgo cardiovascular en mujeres adultas mediante ejercicio físico de sobrecarga. Revista Médica de Chile. 2015; 143 (3): 289-296. doi:10.4067/s0034-98872015000300002

26. Roselló M, Guzmán S, Bolaños M. Efecto de un programa de rehabilitación cardiaca en la alimentación, peso corporal, perfil lipídico y ejercicio físico de pacientes con enfermedad coronaria. Revista Costarricense de Cardiología. 2011; 3 (2): 15-20. 
27. Wong M, García M, García A, Carillo S. Results from the phase II cardiac rehabilitation program, developed by the National Rehabilitation Center, Costa Rica. Acta Med Costarric. 2011; 53: 4.

28. Mancilla R, Torres P, Álvarez C, Schifferli I, Sapunar J, Díaz E. Ejercicio físico interválico de alta intensidad mejora el control glicémico y la capacidad aeróbica en pacientes con intolerancia a la glucosa. Revista Médica de Chile. 2014; 142 (1): 34-39. doi:10.4067/s003498872014000100006.

29. Kodama S, Tanaka S, Saito K, Shu M, Sone Y. Effect of aerobic exercise training on serum levels of high-density lipoprotein cholesterol: a metaanalysis. Arch Intern Med. 2007; 167: 999-1008.
30. Kelley G, Kelley K. Aerobic exercise and HDL2-C: a meta-analysis of randomized controlled trials. Atherosclerosis. 2006; 184: 207-15.

Financiamiento: No se recibió financiamiento de ninguna entidad.

Conflicto de intereses: Los autores declaran no tener ningún tipo de conflicto de intereses. 